SLAC-PUB-13875

\title{
Light-Front Quantization Approach to the Gauge-Gravity Correspondence and Hadron Spectroscopy
}

\author{
Guy F. de Téramond ${ }^{a}$ and Stanley J. Brodsky, ${ }^{b}$ \\ ${ }^{a}$ Universidad de Costa Rica, San José, Costa Rica \\ ${ }^{b}$ SLAC National Accelerator Laboratory, Stanford University, Stanford, CA 94309, USA
}

\begin{abstract}
We find a correspondence between semiclassical QCD quantized on the lightfront and a dual gravity model in anti-de Sitter (AdS) space, thus providing an initial approximation to QCD in its strongly coupled regime. This correspondence - light-front holography - leads to a light-front Hamiltonian and relativistic bound-state wave equations that are functions of an invariant impact variable $\zeta$ which measures the separation of the quark and gluonic constituents within hadrons at equal light-front time. The eigenvalues of the resulting light-front Schrödinger and Dirac equations are consistent with the observed light meson and baryon spectrum, and the eigenmodes provide the light-front wavefunctions, the probability amplitudes describing the dynamics of the hadronic constituents. The light-front equations of motion, which are dual to an effective classical gravity theory, possess remarkable algebraic and integrability properties which are dictated by the underlying conformal properties of the theory. We extend the algebraic construction to include a confining potential while preserving the integrability of the mesonic and baryonic bound-state equations.
\end{abstract}




\section{Introduction}

Quantum Chromodynamics (QCD) provides a fundamental description of hadrons in terms of structureless quarks and gluons. Despite the successful applications of QCD in the perturbative regime, it still remains one of the most difficult field-theoretic problems in strong interaction dynamics to understand the interactions and composition of hadrons in terms of the confined constituent degrees of freedom of the QCD Lagrangian. Thus an important theoretical goal is to find an initial approximation to QCD which is analytically tractable and which can be systematically improved. For example, in quantum electrodynamics, the Coulombic Schrödinger and Dirac equations provide accurate first approximations to atomic bound state problems which can then be systematically improved using the Bethe-Salpeter formalism and correcting for quantum fluctuations, such as the Lamb Shift and vacuum polarization.

The AdS/CFT correspondence [1] between string states on anti-de Sitter (AdS) space-time and conformal gauge field theories (CFT) in physical space-time has brought a new set of tools for studying the dynamics of strongly coupled quantum field theories, and it has led to new analytical insights into the confining dynamics of QCD which is difficult to realize using other methods. The AdS/CFT duality provides a gravity description in a $(d+1)$-dimensional AdS space-time in terms of a flat $d$ dimensional conformally-invariant quantum field theory defined at the AdS asymptotic boundary. 2] Thus, in principle, one can compute physical observables in a strongly coupled gauge theory in terms of a classical gravity theory.

As first shown by Polchinski and Strassler, 3] the AdS/CFT duality, modified to incorporate a mass scale, provides a derivation of dimensional counting rules [4] for the leading power-law fall-off of hard scattering beyond the perturbative regime. The modified theory generates the hard behavior expected from QCD, instead of the soft behavior characteristic of strings. In order to describe a confining theory, the conformal invariance of $\mathrm{AdS}_{5}$ must be broken. A simple way to impose confinement and discrete normalizable modes is to truncate the regime where the string modes can propagate by introducing an infrared (IR) cutoff at a finite value $z_{0} \sim 1 / \Lambda_{\mathrm{QCD}}$. Thus the "hardwall" at $z_{0}$ breaks conformal invariance and allows the introduction of the QCD scale and a spectrum of particle states. [3] The mechanisms of confinement are encoded in the warp of the $\mathrm{AdS}_{5}$ metrics near a large infrared value $z_{0}$ which sets the scale of the strong interactions. In this simplified approach the propagation of hadronic modes in a fixed effective gravitational background encodes salient properties of the QCD dual theory, such as the ultraviolet (UV) conformal limit at the AdS boundary at $z \rightarrow 0$, as well as modifications of the background geometry in the large $z$ infrared region as are characteristic of strings dual to confining gauge theories. Since the AdS metric

$$
d s^{2}=\frac{R^{2}}{z^{2}}\left(\eta_{\mu \nu} d x^{\mu} d x^{\nu}-d z^{2}\right)
$$

is invariant under a dilatation of all coordinates $x^{\mu} \rightarrow \lambda x^{\mu}, z \rightarrow \lambda z$, the variable $z$ acts 
like a scaling variable in Minkowski space: different values of $z$ correspond to different energy scales at which the hadron is examined.

In the usual AdS/QCD approach [5, 6] bulk fields are introduced to match the $S U(2)_{L} \times S U(2)_{R}$ chiral symmetries of QCD and its spontaneous breaking, but without explicit connection with the internal constituent structure of hadrons. [7] Instead, axial and vector currents become the primary entities as in effective chiral theory. Following this bottom-up approach only a limited number of operators are introduced, and consequently only a limited number of fields are required to construct phenomenologically viable five-dimensional gravity duals. One can also effectively modify the metric of AdS space by introducing a dilaton field to reproduce the observed linear Regge behavior of the hadronic spectrum. [8] The resulting model is equivalent to the introduction of a harmonic oscillator confining potential $U \sim \kappa^{4} z^{2}$. In this "soft-wall" model, the value of $\kappa$ breaks conformal invariance, and it sets the mass scale for the hadronic spectrum. The soft-wall background approaches asymptotically AdS space for small values of the variable $z$, as required by the near-conformal limit of QCD in the ultraviolet region.

We have recently shown a remarkable connection between the description of hadronic modes in AdS space and the Hamiltonian formulation of QCD in physical space-time quantized on the light-front (LF) at equal light-front time $\tau$. Light-front quantization is the ideal framework for describing the structure of hadrons in terms of their quark and gluon degrees of freedom. The simple structure of the light-front vacuum allows an unambiguous definition of the partonic content of a hadron in QCD and of hadronic light-front wavefunctions which relate its quark and gluon degrees of freedom to their asymptotic hadronic state. The light-front wavefunctions (LFWFs) of relativistic bound states in QCD provide a fundamental description of the structure and internal dynamics of hadronic states in terms of their constituent quark and gluons at a fixed light-front time $\tau=t+z / c$, the time marked by the front of a light wave, [9]rather than at instant time $t$, the ordinary time. Unlike instant time quantization, the Hamiltonian equation of motion in the LF is frame independent. This makes a direct connection of QCD with AdS/CFT methods possible.

An important first step for establishing the correspondence with AdS space is to observe that the LF bound state Hamiltonian equation of motion in QCD has an essential dependence in the invariant transverse variable $\zeta$, [10] which measures the separation of the quark and gluonic constituents within the hadron at the same LF time. The variable $\zeta$ plays the role of the radial coordinate $r$ in atomic systems. The result is a single-variable light-front relativistic Schrödinger equation. This first approximation to relativistic QCD bound-state systems is equivalent to the equations of motion that describe the propagation of spin- $J$ modes in a fixed gravitational background asymptotic to AdS space. [10] The eigenvalues of the LF Schrödinger equation give the hadronic spectrum and its eigenmodes represent the probability amplitudes of the hadronic constituents. By using the correspondence between $\zeta$ in the LF theory and $z$ in AdS space, one can identify the terms in the dual gravity AdS equations that correspond 
to the kinetic energy terms of the partons inside a hadron and the interaction terms that build confinement. [10] The identification of orbital angular momentum of the constituents in the light-front description is also a key element in our description of the internal structure of hadrons using holographic principles. This mapping was originally obtained by matching the expression for electromagnetic current matrix elements in AdS space with the corresponding expression for the current matrix element using LF theory in physical space time. [11] More recently we have shown that one obtains the identical holographic mapping using the matrix elements of the energy-momentum tensor, [12 thus providing a consistency test and verification of holographic mapping from AdS to physical observables defined on the light front.

The integrability of the equations underlying a physical system is related to its symmetries. As we shall show, conformal symmetry in fact yields integrability: the differential equations describing the relativistic bound state system can be expressed in terms of ladder operators, and the solutions can be written in terms of analytic functions. The application of ladder operators [13] is particularly useful in the study of AdS/QCD models [14] and the description of arbitrary spin fields in AdS space. [15] We will show how the algebraic conformal properties can be extended to include the mass scale of the soft-wall model and thus confinement. This algebraic construction is also extended to build models of baryons.

\section{Light-Front Quantization of QCD}

One can express the hadron four-momentum generator $P=\left(P^{+}, P^{-}, \mathbf{P}_{\perp}\right), P^{ \pm}=P^{0} \pm$ $P^{3}$, in terms of the dynamical fields, the Dirac field $\psi_{+}$, where $\psi_{ \pm}=\Lambda_{ \pm} \psi, \Lambda_{ \pm}=\gamma^{0} \gamma^{ \pm}$, and the transverse field $\mathbf{A}_{\perp}$ in the $A^{+}=0$ gauge [16] quantized on the light-front at fixed light-cone time $x^{+}, x^{ \pm}=x^{0} \pm x^{3}$

$$
\begin{aligned}
P^{-} & =\frac{1}{2} \int d x^{-} d^{2} \mathbf{x}_{\perp} \bar{\psi}_{+} \gamma^{+} \frac{\left(i \nabla_{\perp}\right)^{2}+m^{2}}{i \partial^{+}} \psi_{+}+(\text {interactions }) \\
P^{+} & =\int d x^{-} d^{2} \mathbf{x}_{\perp} \bar{\psi}_{+} \gamma^{+} i \partial^{+} \psi_{+} \\
\mathbf{P}_{\perp} & =\frac{1}{2} \int d x^{-} d^{2} \mathbf{x}_{\perp} \bar{\psi}_{+} \gamma^{+} i \nabla_{\perp} \psi_{+}
\end{aligned}
$$

where the integrals are over the initial surface $x^{+}=0$. The LF Hamiltonian $P^{-}$ generates LF time translations $\left[\psi_{+}(x), P^{-}\right]=i \frac{\partial}{\partial x^{+}} \psi_{+}(x)$, whereas the LF longitudinal $P^{+}$and transverse momentum $\mathbf{P}_{\perp}$ are kinematical generators. As in non-relativistic quantum mechanics, the LF Hamiltonian is additive in the kinetic and potential energy. For simplicity we have omitted from (2- 4) the contributions from the gluon field $\mathbf{A}_{\perp}$.

A physical hadron in four-dimensional Minkowski space has four-momentum $P_{\mu}$ and invariant hadronic mass states $P_{\mu} P^{\mu}=M^{2}$ determined by the Lorentz-invariant 
Hamiltonian equation for the relativistic bound-state system

$$
P_{\mu} P^{\mu}|\psi(P)\rangle=\left(P^{-} P^{+}-\mathbf{P}_{\perp}^{2}\right)|\psi(P)\rangle=M^{2}|\psi(P)\rangle,
$$

where the hadronic state $|\psi\rangle$ is an expansion in multiparticle Fock eigenstates $|n\rangle$ of the free light-front Hamiltonian: $|\psi\rangle=\sum_{n} \psi_{n}|\psi\rangle$. The state $\left|\psi\left(P^{+}, \mathbf{P}_{\perp}, J^{z}\right)\right\rangle$ is an eigenstate of the total momentum $P^{+}$and $\mathbf{P}_{\perp}$ and the total spin $J^{z}$. Quark and gluons appear from the light-front quantization of the excitations of the dynamical fields $\psi_{+}$ and $\mathbf{A}_{\perp}$ expanded in terms of creation and annihilation operators at fixed LF time $\tau$. The Fock components $\psi_{n}\left(x_{i}, \mathbf{k}_{\perp i}, \lambda_{i}^{z}\right)$ are independent of $P^{+}$and $\mathbf{P}_{\perp}$ and depend only on relative partonic coordinates: the momentum fraction $x_{i}=k_{i}^{+} / P^{+}$, the transverse momentum $\mathbf{k}_{\perp i}$ and spin component $\lambda_{i}^{z}$. Momentum conservation requires $\sum_{i=1}^{n} x_{i}=1$ and $\sum_{i=1}^{n} \mathbf{k}_{\perp i}=0$. The LFWFs $\psi_{n}$ provide a frame-independent representation of a hadron which relates its quark and gluon degrees of freedom to their asymptotic hadronic state.

\subsection{A Semiclassical Approximation to QCD}

We can compute $M^{2}$ from the hadronic matrix element

$$
\left\langle\psi\left(P^{\prime}\right)\left|P_{\mu} P^{\mu}\right| \psi(P)\right\rangle=M^{2}\left\langle\psi\left(P^{\prime}\right) \mid \psi(P)\right\rangle,
$$

expanding the initial and final hadronic states in terms of its Fock components. The computation is simplified in the frame $P=\left(P^{+}, M^{2} / P^{+}, \overrightarrow{0}_{\perp}\right)$ where $P^{2}=P^{+} P^{-}$. We find

$$
M^{2}=\sum_{n} \int\left[d x_{i}\right]\left[d^{2} \mathbf{k}_{\perp i}\right] \sum_{q}\left(\frac{\mathbf{k}_{\perp q}^{2}+m_{q}^{2}}{x_{q}}\right)\left|\psi_{n}\left(x_{i}, \mathbf{k}_{\perp i}\right)\right|^{2}+(\text { interactions }),
$$

plus similar terms for antiquarks and gluons $\left(m_{g}=0\right)$. The integrals in (7) are over the internal coordinates of the $n$ constituents for each Fock state

$$
\int\left[d x_{i}\right] \equiv \prod_{i=1}^{n} \int d x_{i} \delta\left(1-\sum_{j=1}^{n} x_{j}\right), \quad \int\left[d^{2} \mathbf{k}_{\perp i}\right] \equiv \prod_{i=1}^{n} \int \frac{d^{2} \mathbf{k}_{\perp i}}{2(2 \pi)^{3}} 16 \pi^{3} \delta^{(2)}\left(\sum_{j=1}^{n} \mathbf{k}_{\perp j}\right),
$$

with phase space normalization $\sum_{n} \int\left[d x_{i}\right]\left[d^{2} \mathbf{k}_{\perp i}\right]\left|\psi_{n}\left(x_{i}, \mathbf{k}_{\perp i}\right)\right|^{2}=1$.

The LFWF $\psi_{n}\left(x_{i}, \mathbf{k}_{\perp i}\right)$ can be expanded in terms of $n-1$ independent position coordinates $\mathbf{b}_{\perp j}, j=1,2, \ldots, n-1$, conjugate to the relative coordinates $\mathbf{k}_{\perp i}$, with $\sum_{i=1}^{n} \mathbf{b}_{\perp i}=0$. We can also express (7) in terms of the internal impact coordinates $\mathbf{b}_{\perp j}$ with the result

$$
\left.M^{2}=\sum_{n} \prod_{j=1}^{n-1} \int d x_{j} d^{2} \mathbf{b}_{\perp j} \psi_{n}^{*}\left(x_{j}, \mathbf{b}_{\perp j}\right) \sum_{q}\left(\frac{-\nabla_{\mathbf{b}_{\perp q}}^{2}+m_{q}^{2}}{x_{q}}\right) \psi_{n}\left(x_{j}, \mathbf{b}_{\perp j}\right)+\text { (interactions }\right) .
$$


The normalization is defined by $\sum_{n} \prod_{j=1}^{n-1} \int d x_{j} d^{2} \mathbf{b}_{\perp j}\left|\psi_{n}\left(x_{j}, \mathbf{b}_{\perp j}\right)\right|^{2}=1$. To simplify the discussion we will consider a two-parton hadronic bound state. In the limit of zero quark mass $m_{q} \rightarrow 0$

$$
M^{2}=\int_{0}^{1} \frac{d x}{x(1-x)} \int d^{2} \mathbf{b}_{\perp} \psi^{*}\left(x, \mathbf{b}_{\perp}\right)\left(-\nabla_{\mathbf{b}_{\perp}}^{2}\right) \psi\left(x, \mathbf{b}_{\perp}\right)+(\text { interactions }) .
$$

The functional dependence for a given Fock state is given in terms of the invariant mass

$$
M_{n}^{2}=\left(\sum_{a=1}^{n} k_{a}^{\mu}\right)^{2}=\sum_{a} \frac{\mathbf{k}_{\perp a}^{2}+m_{a}^{2}}{x_{a}} \rightarrow \frac{\mathbf{k}_{\perp}^{2}}{x(1-x)},
$$

giving the measure of the off-energy shell of the bound state, $M^{2}-M_{n}^{2}$. Similarly in impact space the relevant variable for a two-parton state is $\zeta^{2}=x(1-x) \mathbf{b}_{\perp}^{2}$. Thus, to first approximation LF dynamics depend only on the boost invariant variable $M_{n}$ or $\zeta$, and hadronic properties are encoded in the hadronic mode $\phi(\zeta)$ from the relation

$$
\psi(x, \zeta, \varphi)=e^{i L \varphi} X(x) \frac{\phi(\zeta)}{\sqrt{2 \pi \zeta}}
$$

thus factoring out the angular dependence $\varphi$ and the longitudinal, $X(x)$, and transverse mode $\phi(\zeta)$ with normalization $\langle\phi \mid \phi\rangle=\int d \zeta|\langle\zeta \mid \phi\rangle|^{2}=1$.

We can write the Laplacian operator in (10) in circular cylindrical coordinates $(\zeta, \varphi)$ and factor out the angular dependence of the modes in terms of the $S O(2)$ Casimir representation $L^{2}$ of orbital angular momentum in the transverse plane. Using (12) we find [10]

$$
M^{2}=\int d \zeta \phi^{*}(\zeta) \sqrt{\zeta}\left(-\frac{d^{2}}{d \zeta^{2}}-\frac{1}{\zeta} \frac{d}{d \zeta}+\frac{L^{2}}{\zeta^{2}}\right) \frac{\phi(\zeta)}{\sqrt{\zeta}}+\int d \zeta \phi^{*}(\zeta) U(\zeta) \phi(\zeta),
$$

where all the complexity of the interaction terms in the QCD Lagrangian is summed in the effective potential $U(\zeta)$. The LF eigenvalue equation $P_{\mu} P^{\mu}|\phi\rangle=M^{2}|\phi\rangle$ is thus a light-front wave equation for $\phi$

$$
\left(-\frac{d^{2}}{d \zeta^{2}}-\frac{1-4 L^{2}}{4 \zeta^{2}}+U(\zeta)\right) \phi(\zeta)=M^{2} \phi(\zeta)
$$

a relativistic single-variable LF Schrödinger equation. Its eigenmodes $\phi(\zeta)=\langle\zeta \mid \phi\rangle$ determine the hadronic mass spectrum and represent the probability amplitude to find $n$-partons at transverse impact separation $\zeta$, the invariant separation between pointlike constituents within the hadron [11] at equal LF time. Extension of the results to arbitrary $n$ follows from the $x$-weighted definition of the transverse impact variable of the $n-1$ spectator system [11]: $\zeta=\sqrt{\frac{x}{1-x}}\left|\sum_{j=1}^{n-1} x_{j} \mathbf{b}_{\perp j}\right|$, where $x=x_{n}$ is the longitudinal momentum fraction of the active quark. One can also generalize the equations to allow for the kinetic energy of massive quarks using Eqs. (7) or (9). In this case, however, the longitudinal mode $X(x)$ does not decouple from the effective LF bound-state equations. 


\section{Mesons in Light-Front Holography}

On AdS space the physical states are represented by normalizable modes $\Phi_{P}\left(x^{\mu}, z\right)=$ $e^{-i P \cdot x} \Phi(z)$, with plane waves along the Poncaré coordinates and a profile function $\Phi(z)$ along the holographic coordinate $z$. Each LF hadronic state $|\psi(P)\rangle$ is dual to a normalizable string mode $\Phi_{P}\left(x^{\mu}, z\right)$ and the hadronic mass $M^{2}$ is found by solving the eigenvalue problem for the corresponding wave equation in AdS space, which, as we show below, is equivalent to the semiclassical approximation Eq. (14).

As the simplest example we consider a truncated model where quark and gluons propagate freely in the hadronic interior and the interaction terms effectively build the confinement. The effective potential is a hard wall: $U(\zeta)=0$ if $\zeta \leq 1 / \Lambda_{\mathrm{QCD}}$ and $U(z)=\infty$ if $\zeta>1 / \Lambda_{\mathrm{QCD}}$. This provides an analog of the MIT bag model where quarks are permanently confined inside a finite region of space. [17] However, unlike bag models, boundary conditions are imposed on the boost invariant variable $\zeta$, not on the bag radius at fixed time. The resulting model is a manifestly Lorentz invariant model with confinement at large distances and conformal behavior at short distances. Confinement is imposed with the boundary conditions $\phi\left(\zeta=1 / \Lambda_{\mathrm{QCD}}\right)=0$.

To study the stability properties of the quantum-mechanical system, it is convenient to consider a continuous parameter $\nu$ instead of $L$ which has quantized values. If $\nu^{2} \geq 0$ the LF Hamiltonian $P_{\mu} P^{\mu}$ in the $\zeta$-representation

$$
H_{L F}^{\nu}(\zeta)=-\frac{d^{2}}{d \zeta^{2}}-\frac{1-4 \nu^{2}}{4 \zeta^{2}}
$$

is written as a bilinear form

$$
H_{L F}^{\nu}(\zeta)=\Pi_{\nu}^{\dagger}(\zeta) \Pi_{\nu}(\zeta), \quad \nu^{2} \geq 0
$$

where the operator $\Pi_{\nu}$ and its adjoint $\Pi_{\nu}^{\dagger}$ are defined by

$$
\Pi_{\nu}(\zeta)=-i\left(\frac{d}{d \zeta}-\frac{\nu+\frac{1}{2}}{\zeta}\right), \quad \Pi_{\nu}^{\dagger}(\zeta)=-i\left(\frac{d}{d \zeta}+\frac{\nu+\frac{1}{2}}{\zeta}\right)
$$

with the commutation relation

$$
\left[\Pi_{\nu}(\zeta), \Pi_{\nu}^{\dagger}(\zeta)\right]=\frac{2 \nu+1}{\zeta^{2}}
$$

For $\nu^{2} \geq 0$ the Hamiltonian is positive definite, $\left\langle\phi\left|H_{L C}^{\nu}\right| \phi\right\rangle=\int d \zeta\left|\Pi_{\nu} \phi(z)\right|^{2} \geq$ 0 , and consequently $M^{2} \geq 0$. For $\nu^{2}<0$ the Hamiltonian cannot be written as a bilinear product and the Hamiltonian is unbounded from below. The particle "falls towards the center" [18]. The critical value corresponds to $\nu=0$. Thus, if $\nu^{2}<0$ the expectation values of the Hamiltonian are negative and $M^{2}<0$. The critical value $\nu=0$ corresponds the the lowest possible stable solution which we identify with the ground state of the LF Hamiltonian and thus with zero orbital angular momentum 
$L=0$. Higher orbital excitations are constructed by the $L$-th application of the raising operator on the ground state

$$
\left\langle\zeta \mid \phi_{L}\right\rangle=C_{L} \sqrt{\zeta}(-\zeta)^{L}\left(\frac{1}{\zeta} \frac{d}{d \zeta}\right)^{L} J_{0}(\zeta M)=C_{L} \sqrt{\zeta} J_{L}(\zeta M) .
$$

The solutions $\phi_{L}(\zeta)=\left\langle\zeta \mid \phi_{L}\right\rangle$ are eigenfunctions of the LF equation (14) with $U=0$ and quantized orbital excitations, $L=0,1,2, \cdots$. We thus recover the Casimir form $L^{2}$ corresponding to the $S O(2)$ group of rotations in the transverse LF plane. The mode spectrum follows from the boundary conditions at $\zeta=1 / \Lambda_{\mathrm{QCD}}$, and it is given in terms of the roots of the Bessel functions: $M_{L, k}=\beta_{L, k} \Lambda_{\mathrm{QCD}}$. For large quantum numbers $M \sim 2 n+L$, in contrast to the usual Regge dependence $M^{2} \sim n+L$.

Upon the substitution $\Phi(\zeta) \sim \zeta^{3 / 2} \phi(\zeta), \zeta \rightarrow z$, in the light-front wave equation (14) for $U=0$ we find

$$
\left[z^{2} \partial_{z}^{2}-3 z \partial_{z}+z^{2} M^{2}-(\mu R)^{2}\right] \Phi(z)=0
$$

the wave equation which describes the propagation of scalar modes in $\mathrm{AdS}_{5}$ space with AdS radius $R$. The five-dimensional mass $\mu$ is related to the orbital angular momentum of the hadronic bound state by $(\mu R)^{2}=-4+L^{2}$. The quantum-mechanical stability condition $L^{2} \geq 0$ is thus equivalent to the AdS Breitenlohner-Freedman stability bound [19] $(\mu R)^{2} \geq-4$. Since in the conformal limit $U \rightarrow 0$, Eq. (14) is equivalent to an AdS wave equation for the scalar field $\Phi(z)$. The hard-wall light-front model discussed here is thus equivalent to the hard wall model of Ref. [3].

Each hadronic state of spin $J$ is dual to a normalizable mode $\Phi_{P}\left(x^{\mu}, z\right)_{\mu_{1} \cdots \mu_{J}}=$ $e^{-i P \cdot x} \Phi(z)_{\mu_{1} \cdots \mu_{J}}$, with all the (totally symmetric) polarization indices chosen along the $3+1$ Poincaré coordinates. One can thus construct an effective action in terms of high spin modes with only the physical degrees of freedom $\Phi\left(x^{\mu}, z\right)_{\mu_{1} \cdots \mu_{J}}$ by shifting dimensions $\Phi_{J}(z)=(z / R)^{-J} \Phi(z)$. The shifted field $\Phi_{J}$ obeys the equation of motion

$$
\left[z^{2} \partial_{z}^{2}-(3-2 J) z \partial_{z}+z^{2} M^{2}-(\mu R)^{2}\right] \Phi_{J}=0
$$

where the fifth dimensional mass is rescaled according to $(\mu R)^{2} \rightarrow(\mu R)^{2}-J(4-$ $J)$. Having written the wave equation for a $J$-mode in AdS space, we can find the corresponding equation in physical $3+1$ space by performing the substitution $z \rightarrow \zeta$ and $\phi_{J}(\zeta) \sim \zeta^{-3 / 2+J} \Phi_{J}(\zeta)$. We find

$$
\left(-\frac{d^{2}}{d \zeta^{2}}-\frac{1-4 L^{2}}{4 \zeta^{2}}\right) \phi_{\mu_{1} \cdots \mu_{J}}=M^{2} \phi_{\mu_{1} \cdots \mu_{J}}
$$

where $\mu$ is not a free parameter but scales according to $(\mu R)^{2}=-(2-J)^{2}+L^{2}$. In the hard-wall model there is a total decoupling of the total spin $J$ and the $\pi$ and $\rho$ mesons are degenerate. The scaling dimensions are $\Delta=2+L$ independent of $J$ in agreement with the twist scaling dimension of a two parton bound state in QCD. 


\subsection{A Soft-Wall Light-Front Model for Mesons}

The conformal algebraic structure of the hard wall model can be extended to include a scale $\kappa$. This procedure breaks conformal invariance and provides a solution for the confinement of modes, while maintaining an integrable algebraic structure. It also allows one to determine the stability conditions for the solutions. The resulting model resembles the soft wall model of Ref. [8]. We write the bound-state LF Hamiltonian as a bilinear product of operators plus a constant $C\left(\kappa^{2}\right)$ to be determined:

$$
H_{L F}^{\nu}(\zeta)=\Pi_{\nu}^{\dagger}(\zeta) \Pi_{\nu}(\zeta)+C, \quad \nu^{2} \geq 0
$$

where the LF generator $\Pi$ and its adjoint $\Pi^{\dagger}$

$$
\Pi_{\nu}(\zeta)=-i\left(\frac{d}{d \zeta}-\frac{\nu+\frac{1}{2}}{\zeta}-\kappa^{2} \zeta\right), \quad \Pi_{\nu}^{\dagger}(\zeta)=-i\left(\frac{d}{d \zeta}+\frac{\nu+\frac{1}{2}}{\zeta}+\kappa^{2} \zeta\right)
$$

obey the commutation relation

$$
\left[\Pi_{\nu}(\zeta), \Pi_{\nu}^{\dagger}(\zeta)\right]=\frac{2 \nu+1}{\zeta^{2}}-2 \kappa^{2} .
$$

For $\nu^{2} \geq 0$ and $C \geq-4 \kappa^{2}$, the Hamiltonian is positive definite, $\left\langle\phi\left|H_{L C}^{\nu}\right| \phi\right\rangle,=$ $\int d \zeta\left|\Pi_{\nu} \phi(z)\right|^{2}+C \geq 0$ and $M^{2} \geq 0$. For $\nu^{2}<0$ the Hamiltonian cannot be written as a bilinear product and the Hamiltonian is unbounded from below. The lowest stable solution of the extended LF Hamiltonian corresponds to $C=-4 \kappa^{2}$ and $\nu=0$ and it is massless, $M^{2}=0$. We impose chiral symmetry by choosing $C=-4 \kappa^{2}$ and thus identifying the ground state with the pion. With this choice of the constant $C$, the LF Hamiltonian (22) is

$$
H_{L F}(\zeta)=-\frac{d^{2}}{d \zeta^{2}}-\frac{1-4 L^{2}}{4 \zeta^{2}}+\kappa^{4} \zeta^{2}+2 \kappa^{2}(L-1)
$$

with eigenfunctions

$$
\phi_{L}(\zeta)=\kappa^{1+L} \sqrt{\frac{2 n !}{(n+L) !}} \zeta^{1 / 2+L} e^{-\kappa^{2} \zeta^{2} / 2} L_{n}^{L}\left(\kappa^{2} \zeta^{2}\right)
$$

and eigenvalues $\mathcal{M}^{2}=4 \kappa^{2}(n+L)$. This is illustrated in Fig. 1 for the pseudoscalar meson spectra.

Next we show that the confining model has also an effective classical gravity description corresponding to an $\mathrm{AdS}_{5}$ geometry modified by a positive-sign dilaton background $\exp \left(+\kappa^{2} z^{2}\right)$, with sign opposite to that of reference of Ref. [8]. The positive dilaton solution has interesting physical implications, since it leads to a confining potential between heavy quarks [21] and to a convenient framework for describing chiral symmetry breaking. [22] It also leads to the identification of a nonperturbative effective strong 

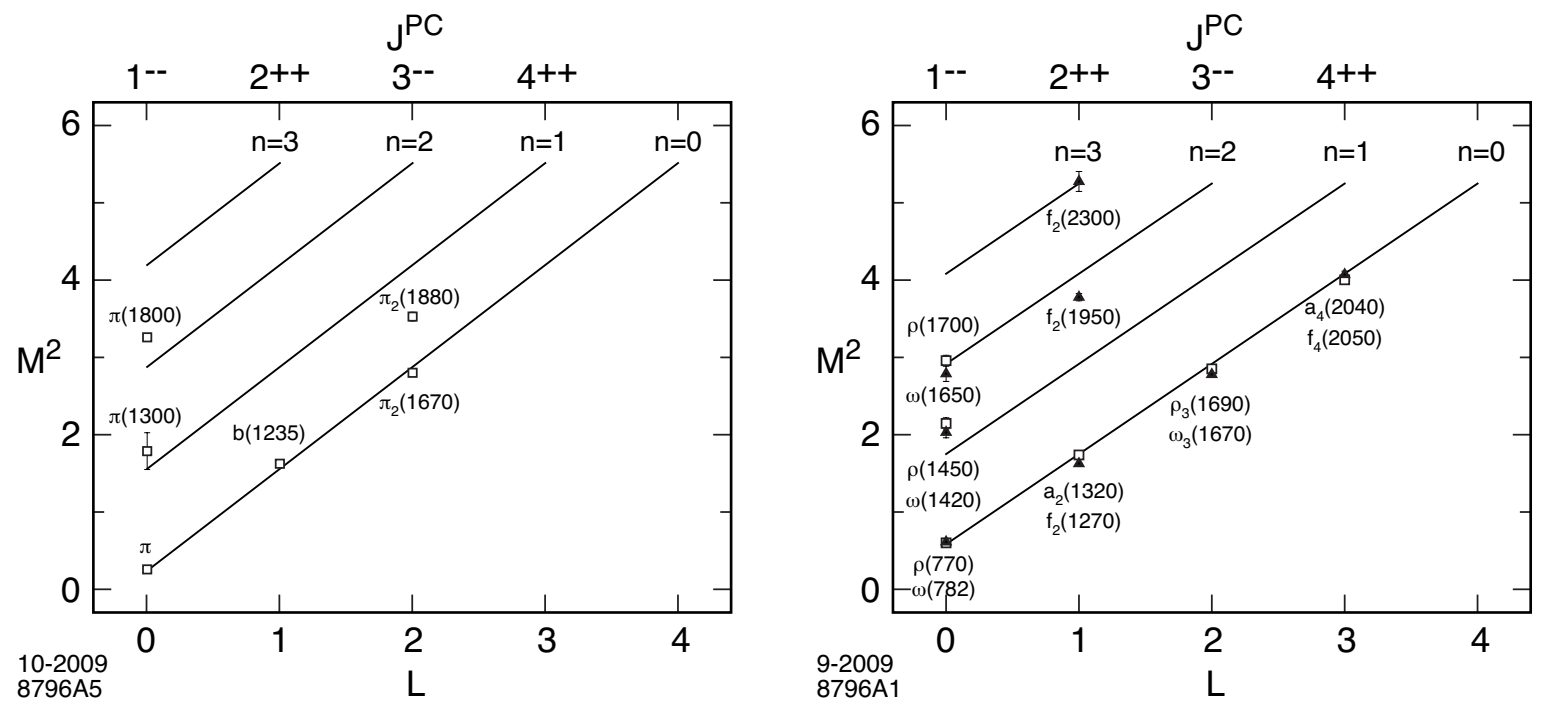

Figure 1: Parent and daughter Regge trajectories for the $\pi$-meson family for $\kappa=0.6 \mathrm{GeV}$ (left) and for the $I=1 \rho$-meson and the $I=0 \omega$-meson families for $\kappa=0.54 \mathrm{GeV}$ (right). The data is from [20].

coupling $\alpha_{s}$ and $\beta$-functions which are in agreement with available data and lattice simulations. 23] In presence of a dilaton profile $\exp \left(+\kappa^{2} z^{2}\right)$ the wave equation for a spin $J$ mode $\Phi(z)_{\mu_{1} \cdots \mu_{J}}$ is given by [24]

$$
\left[z^{2} \partial_{z}^{2}-\left(3-2 J-2 \kappa^{2} z^{2}\right) z \partial_{z}+z^{2} M^{2}-(\mu R)^{2}\right] \Phi_{J}=0
$$

and reduces to (20) in the conformal limit $\kappa \rightarrow 0$. Upon the substitution $z \rightarrow \zeta$ and $\phi_{J}(\zeta) \sim \zeta^{-3 / 2+J} e^{\kappa^{2} \zeta^{2} / 2} \Phi_{J}(\zeta)$, we find the $\mathrm{LF}$ wave equation

$$
\left(-\frac{d^{2}}{d \zeta^{2}}-\frac{1-4 L^{2}}{4 \zeta^{2}}+\kappa^{4} \zeta^{2}+2 \kappa^{2}(L+S-1)\right) \phi_{\mu_{1} \cdots \mu_{J}}=M^{2} \phi_{\mu_{1} \cdots \mu_{J}}
$$

with $J_{z}=L_{z}+S_{z}$ and $(\mu R)^{2}=-(2-J)^{2}+L^{2}$. Equation (28) has eigenfunctions given by (26) and eigenvalues $M_{n, L, S}^{2}=4 \kappa^{2}(n+L+S / 2)$. The results for $S=1$ vector mesons is illustrated in Fig. 1, where the spectrum is built by simply adding $4 \kappa^{2}$ for a unit change in the radial quantum number, $4 \kappa^{2}$ for a change in one unit in the orbital quantum number and $2 \kappa^{2}$ for a change of one unit of spin to the ground state value of $M^{2}$. Remarkably, the same rule holds for baryons as shown below.

\section{Baryons in Light-Front Holography}

The effective light-front wave equation which describes baryonic states in holographic QCD is a linear equation determined by the LF transformation properties of spin 1/2 states. We write

$$
D_{L F}(\zeta) \psi(\zeta)=M \psi(\zeta)
$$


where $D_{L F}$ is a hermitian operator, $D_{L F}=D_{L F}^{\dagger}$, thus $D_{L F}^{2}=M^{2}$. We write $D_{L F}$ as a product $D_{L F}=\alpha \Pi$, where $\Pi$ is the matrix valued (non-hermitian) generator

$$
\Pi_{\nu}(\zeta)=-i\left(\frac{d}{d \zeta}-\frac{\nu+\frac{1}{2}}{\zeta} \gamma\right)
$$

If follows from the square of $D_{L F}, D_{L F}^{2}=M^{2}$, that the matrices $\alpha$ and $\gamma$ are $4 \times 4$ anti-commuting hermitian matrices with unit square. The operator $\Pi$ and its adjoint $\Pi^{\dagger}$ thus satisfy the commutation relation

$$
\left[\Pi_{\nu}(\zeta), \Pi_{\nu}^{\dagger}(\zeta)\right]=\frac{2 \nu+1}{\zeta^{2}} \gamma
$$

The light front Hamiltonian $H_{L F}$ is

$$
H_{L F}^{\nu}(\zeta)=\Pi_{\nu}(\zeta)^{\dagger} \Pi_{\nu}(\zeta)=-\frac{d^{2}}{d \zeta^{2}}+\frac{\left(\nu+\frac{1}{2}\right)^{2}}{\zeta^{2}}-\frac{\nu+\frac{1}{2}}{\zeta^{2}} \gamma
$$

The LF equation $H_{L F} \psi_{ \pm}=M^{2} \psi_{ \pm}$, has a two-component solution

$$
\psi_{+}(\zeta) \sim \sqrt{\zeta} J_{\nu}(\zeta M), \quad \psi_{-}(\zeta) \sim \sqrt{\zeta} J_{\nu+1}(\zeta M)
$$

where $\gamma \psi_{ \pm}= \pm \psi_{ \pm}$. Thus $\gamma$ is the four dimensional chirality operator $\gamma_{5}$. In the Weyl representation

$$
\gamma=\left(\begin{array}{cc}
I & 0 \\
0 & -I
\end{array}\right) \quad \text { and } \quad i \alpha=\left(\begin{array}{cc}
0 & I \\
-I & 0
\end{array}\right)
$$

The effective LF equation for baryons (29) is indeed equivalent to the Dirac equation describing the propagation of spin-1/2 hadronic modes, on $\operatorname{AdS}_{5}$ space $\Psi_{P}\left(x^{\mu}, z\right)=$ $e^{-i P \cdot x} \Psi(z)$

$$
\left[i\left(z \eta^{\ell m} \Gamma_{\ell} \partial_{m}+2 \Gamma_{z}\right)+\mu R\right] \Psi=0
$$

where $\ell, m$ represent the indices of the full space with coordinates $x^{\mu}$ and $z$. Upon the transformation $\Psi(z) \sim z^{2} \psi(z), z \rightarrow \zeta$, we recover (29) with $\mu R=\nu+1 / 2$ and $\Gamma_{z}=-i \gamma$. Higher spin fermionic modes $\Psi_{\mu_{1} \cdots \mu_{J-1 / 2}}, J>1 / 2$, with all polarization indices along the $3+1$ coordinates follow by shifting dimensions as shown for the case of mesons.

\subsection{A Soft-Wall Light-Front Model for Baryons}

An effective LF equation for baryons with a mass gap $\kappa$ is constructed by extending the conformal algebraic structure for baryons described above, following the analogy with the mesons. We write the effective LF Dirac equation (29) in terms of the matrix-valued operator $\Pi$ and its adjoint $\Pi^{\dagger}$

$$
\Pi_{\nu}(\zeta)=-i\left(\frac{d}{d \zeta}-\frac{\nu+\frac{1}{2}}{\zeta} \gamma-\kappa^{2} \zeta \gamma\right), \quad \Pi_{\nu}^{\dagger}(\zeta)=-i\left(\frac{d}{d \zeta}+\frac{\nu+\frac{1}{2}}{\zeta} \gamma+\kappa^{2} \zeta \gamma\right)
$$




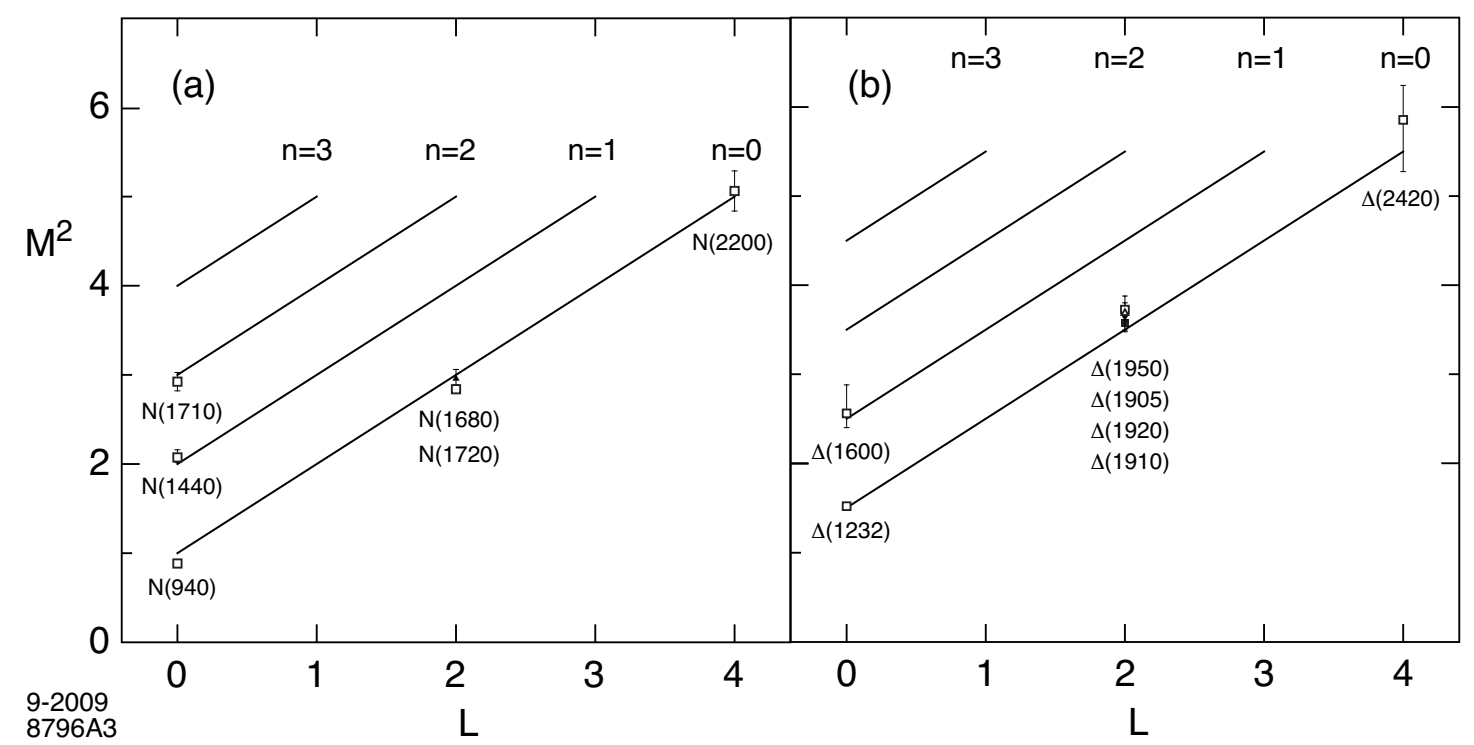

Figure 2: 56 Parent and daughter Regge trajectories for the $N$ and $\Delta$ baryon families for $\kappa=0.5 \mathrm{GeV}$. Data from [20].

with the commutation relation

$$
\left[\Pi_{\nu}(\zeta), \Pi_{\nu}^{\dagger}(\zeta)\right]=\left(\frac{2 \nu+1}{\zeta^{2}}-2 \kappa^{2}\right) \gamma
$$

The extended baryonic model also has a geometric interpretation. It corresponds to the Dirac equation in $\mathrm{AdS}_{5}$ space in presence of a linear potential $\kappa^{2} z$

$$
\left[i\left(z \eta^{\ell m} \Gamma_{\ell} \partial_{m}+2 \Gamma_{z}\right)+\kappa^{2} z+\mu R\right] \Psi=0,
$$

as can be shown directly by using the transformation $\Psi(z) \sim z^{2} \psi(z), z \rightarrow \zeta$.

Before computing the baryon spectrum we must fix the overall scale and the parameter $\nu$. As for the case of the mesons Eq. (22) we write the LF Hamiltonian $H_{L F}^{\nu}=\Pi_{\nu}^{\dagger} \Pi_{\nu}+C$ and chose the same value for $C: C=-4 \kappa^{2}$. With this choice for $C$ the LF Hamiltonian is

$$
H_{L F}=-\frac{d^{2}}{d \zeta^{2}}+\frac{\left(\nu+\frac{1}{2}\right)^{2}}{\zeta^{2}}-\frac{\nu+\frac{1}{2}}{\zeta^{2}} \gamma_{5}+\kappa^{4} \zeta^{2}+\kappa^{2}(2 \nu-3)+\kappa^{2} \gamma_{5}
$$

The LF equation $H_{L F} \psi_{ \pm}=M^{2} \psi_{ \pm}$, has a two-component solution

$$
\psi_{+}(\zeta) \sim z^{\frac{1}{2}+\nu} e^{-\kappa^{2} \zeta^{2} / 2} L_{n}^{\nu}\left(\kappa^{2} \zeta^{2}\right), \quad \psi_{-}(\zeta) \sim z^{\frac{3}{2}+\nu} e^{-\kappa^{2} \zeta^{2} / 2} L_{n}^{\nu+1}\left(\kappa^{2} \zeta^{2}\right)
$$

and eigenvalues $M^{2}=4 \kappa^{2}(n+\nu)$, identical for both plus and minus eigenfunctions.

The baryon interpolating operator $\mathcal{O}_{3+L}=\psi D_{\left\{\ell_{1}\right.} \ldots D_{\ell_{q}} \psi D_{\ell_{q+1}} \ldots D_{\left.\ell_{m}\right\}} \psi, L=$ $\sum_{i=1}^{m} \ell_{i}$, is a twist 3 , dimension $9 / 2+L$ operator with scaling behavior given by its 
twist-dimension $3+L$. We thus require $\nu=L+1$ to match the short distance scaling behavior. Higher spin fermionic modes $\Psi_{\mu_{1} \cdots \mu_{J-1 / 2}}, J>1 / 2$, are obtained by shifting dimensions for the fields as in the bosonic case. Thus, as in the meson sector, the increase in the mass squared for higher baryonic states is $\Delta n=4 \kappa^{2}, \Delta L=4 \kappa^{2}$ and $\Delta S=2 \kappa^{2}$, relative to the lowest ground state, the proton.

The predictions for the 56-plet of light baryons under the $S U(6)$ flavor group are shown in Fig. 2. As for the predictions for mesons in Fig. 1, only confirmed PDG [20] states are shown. The Roper state $N(1440)$ and the $N(1710)$ are well accounted for in this model as the first and second radial states. Likewise the $\Delta(1660)$ corresponds to the first radial state of the $\Delta$ family. The model is successful in explaining the important parity degeneracy observed in the light baryon spectrum, such as the $L=2$, $N(1680)-N(1720)$ pair and the $\Delta(1905), \Delta(1910), \Delta(1920), \Delta(1950)$ states which are degenerate within error bars. The parity degeneracy of baryons is also a property of the hard wall model, but radial states are not well described by this model. [25] For other recent calculations of the hadronic spectrum based on AdS/QCD, see Refs. [26, 27, 28, 29, 30, 31, 32, 33, 34, 35, 36, 37, 38, 39.

\section{Conclusion}

We have derived a correspondence between a semiclassical first approximation to QCD quantized on the light-front and hadronic modes propagating on a fixed AdS background. This provides a duality between the bosonic and fermionic wave equations in AdS higher dimensional space and the corresponding LF equations in physical $3+1$ space. The duality leads to Schrödinger and Dirac-like equations for hadronic bound states in physical space-time when one identifies the AdS fifth dimension coordinate $z$ with the LF coordinate $\zeta$. The light-front equations of motion, which are dual to an effective classical gravity theory, possess remarkable algebraic and integrability properties which follow from the underlying conformal properties of the theory. We also extend the algebraic construction to include a confining potential while preserving the integrability of the mesonic and baryonic bound-state equations.

The light-front holographic theory provides successful predictions for the light-quark meson and baryon spectra, as function of hadron spin, quark angular momentum, and radial quantum number. [40] Using the dilaton framework the pion is massless, corresponding to zero mass quarks, in agreement with chiral invariance arguments.

Higher spin light-front equations can be derived by shifting dimensions in the AdS wave equations. Unlike the top-down string theory approach, one is not limited to hadrons of maximum spin $J \leq 2$, and one can study baryons with finite color $N_{C}=3$.

Both the hard and soft-wall models predict similar multiplicity of states for mesons and baryons as it is observed experimentally. [41] In the hard-wall model the dependence has the form: $M \sim 2 n+L$. However, in the soft-wall model the observed Regge behavior is found: $M^{2} \sim n+L$, which has the same slope in radial quantum number and orbital angular momentum. 
The semiclassical approximation to light-front QCD described in this paper does not account for particle creation and absorption; it is thus expected to break down at short distances where hard gluon exchange and quantum corrections become important. However, one can systematically improve the semiclassical approximation by introducing nonzero quark masses and short-range Coulomb corrections, thus extending the predictions of the model to the dynamics and spectra of heavy and heavy-light quark systems.

\section{Acknowledgments}

Invited plenary talk presented by GdT at Hadron 2009, The International Conference on Hadron Spectroscopy, Florida State University, Tallahassee, November 29 - December 4, 2009. We thank Alexandre Deur, Josh Erlich and Hans-Guenter Dosch for helpful conversations and collaborations. This research was supported by the Department of Energy contract DE-AC02-76SF00515.

\section{References}

[1] J. M. Maldacena, "The large N limit of superconformal field theories and supergravity," Adv. Theor. Math. Phys. 2, 231 (1998) arXiv:hep-th/9711200].

[2] S. S. Gubser, I. R. Klebanov and A. M. Polyakov, "Gauge theory correlators from non-critical string theory," Phys. Lett. B 428, 105 (1998) |arXiv:hep-th/9802109]; E. Witten, "Anti-de Sitter space and holography," Adv. Theor. Math. Phys. 2, 253 (1998) arXiv:hep-th/9802150].

[3] J. Polchinski and M. J. Strassler, "Hard scattering and gauge/string duality," Phys. Rev. Lett. 88, 031601 (2002) |arXiv:hep-th/0109174].

[4] S. J. Brodsky and G. R. Farrar, "Scaling Laws At Large Transverse Momentum," Phys. Rev. Lett. 31, 1153 (1973); V. A. Matveev, R. M. Muradian and A. N. Tavkhelidze, "Automodellism In The Large - Angle Elastic Scattering And Structure Of Hadrons," Lett. Nuovo Cim. 7, 719 (1973).

[5] J. Erlich, E. Katz, D. T. Son and M. A. Stephanov, "QCD and a holographic model of hadrons," Phys. Rev. Lett. 95, 261602 (2005) arXiv:hep-ph/0501128.

[6] L. Da Rold and A. Pomarol, "Chiral symmetry breaking from five dimensional spaces," Nucl. Phys. B 721, 79 (2005) arXiv:hep-ph/0501218.

[7] S. J. Brodsky and G. F. de Teramond, "Light-front hadron dynamics and AdS/CFT correspondence," Phys. Lett. B 582, 211 (2004) arXiv:hep-th/0310227. 
[8] A. Karch, E. Katz, D. T. Son and M. A. Stephanov, "Linear confinement and AdS/QCD," Phys. Rev. D 74, 015005 (2006). [arXiv:hep-ph/0602229].

[9] P. A. M. Dirac, "Forms of Relativistic Dynamics," Rev. Mod. Phys. 21, 392 (1949).

[10] G. F. de Teramond and S. J. Brodsky, "Light-Front Holography: A First Approximation to QCD," Phys. Rev. Lett. 102, 081601 (2009) [arXiv:0809.4899 [hep-ph]].

[11] S. J. Brodsky and G. F. de Teramond, "Hadronic spectra and light-front wavefunctions in holographic QCD," Phys. Rev. Lett. 96, 201601 (2006) |arXiv:hep-ph/0602252]; "Light-Front Dynamics and AdS/QCD Correspondence: The Pion Form Factor in the Space- and Time-Like Regions," Phys. Rev. D 77, 056007 (2008) arXiv:0707.3859 [hep-ph]].

[12] S. J. Brodsky and G. F. de Teramond, "Light-Front Dynamics and AdS/QCD Correspondence: Gravitational Form Factors of Composite Hadrons," Phys. Rev. D 78, 025032 (2008) arXiv:0804.0452 [hep-ph]].

[13] L. Infeld, "On a New Treatment of Some Eigenvalue Problems", Phys. Rev. 59, 737 (1941).

[14] S. J. Brodsky and G. F. de Teramond, "AdS/CFT and Light-Front QCD," arXiv:0802.0514 [hep-ph].

[15] R. R. Metsaev, "Shadows, currents and AdS," Phys. Rev. D 78, 106010 (2008) arXiv:0805.3472 [hep-th]]; "CFT adapted gauge invariant formulation of massive arbitrary spin fields in AdS," Phys. Lett. B 682, 455 (2010) arXiv:0907.2207 [hep-th]].

[16] S. J. Brodsky, H. C. Pauli and S. S. Pinsky, "Quantum chromodynamics and other field theories on the light cone," Phys. Rep. 301, 299 (1998) arXiv:hep-ph/9705477].

[17] A. Chodos, R. L. Jaffe, K. Johnson, C. B. Thorn and V. F. Weisskopf, "A New Extended Model Of Hadrons," Phys. Rev. D 9, 3471 (1974).

[18] L. D. Landau and E. M. Lifshitz, Quantum Mechanics, Pergamon, New York, 1958, Sec. 35.

[19] P. Breitenlohner and D. Z. Freedman, "Stability In Gauged Extended Supergravity," Annals Phys. 144, 249 (1982).

[20] C. Amsler et al. (Particle Data Group), "Review of particle physics," Phys. Lett. B 667 (2008) 1.

[21] O. Andreev, "1/q**2 corrections and gauge / string duality," Phys. Rev. D 73, 107901 (2006) arXiv:hep-th/0603170. 
[22] F. Zuo, "Improved Soft-Wall model with a negative dilaton," arXiv:0909.4240 [hep-ph].

[23] S. J. Brodsky, G. F. de Teramond and A. Deur, to be published.

[24] G. F. de Teramond and S. J. Brodsky, "Light-Front Holography and Gauge/Gravity Duality: The Light Meson and Baryon Spectra," arXiv:0909.3900 [hep-ph].

[25] G. F. de Teramond and S. J. Brodsky, "The hadronic spectrum of a holographic dual of QCD," Phys. Rev. Lett. 94, 201601 (2005) [arXiv:hep-th/0501022].

[26] H. Boschi-Filho and N. R. F. Braga, "Gauge / string duality and scalar glueball mass ratios," JHEP 0305, 009 (2003) arXiv:hep-th/0212207.

[27] H. Boschi-Filho, N. R. F. Braga and H. L. Carrion, "Glueball Regge trajectories from gauge/string duality and the Pomeron," Phys. Rev. D 73, 047901 (2006) arXiv:hep-th/0507063.

[28] N. Evans and A. Tedder, "Perfecting the ultra-violet of holographic descriptions of QCD," Phys. Lett. B 642, 546 (2006) arXiv:hep-ph/0609112.

[29] D. K. Hong, T. Inami and H. U. Yee, "Baryons in AdS/QCD," Phys. Lett. B 646, 165 (2007) arXiv:hep-ph/0609270].

[30] P. Colangelo, F. De Fazio, F. Jugeau and S. Nicotri, "On the light glueball spectrum in a holographic description of QCD," Phys. Lett. B 652, 73 (2007) [arXiv:hep-ph/0703316].

[31] H. Forkel, "Holographic glueball structure," Phys. Rev. D 78, 025001 (2008) arXiv:0711.1179 [hep-ph]].

[32] A. Vega and I. Schmidt, "Scalar hadrons in $A d S_{5} \times S^{5}$," Phys. Rev. D 78 (2008) 017703 [arXiv:0806.2267 [hep-ph]].

[33] K. Nawa, H. Suganuma and T. Kojo, "Baryons with holography," Mod. Phys. Lett. A 23, 2364 (2008) [arXiv:0806.3040 [hep-th]].

[34] W. de Paula, T. Frederico, H. Forkel and M. Beyer, "Dynamical AdS/QCD with area-law confinement and linear Regge trajectories," Phys. Rev. D 79, 075019 (2009) [arXiv:0806.3830 [hep-ph]].

[35] P. Colangelo, F. De Fazio, F. Giannuzzi, F. Jugeau and S. Nicotri, "Light scalar mesons in the soft-wall model of AdS/QCD," Phys. Rev. D 78 (2008) 055009 arXiv:0807.1054 [hep-ph]]. 
[36] H. Forkel and E. Klempt, "Diquark correlations in baryon spectroscopy and holographic QCD," Phys. Lett. B 679, 77 (2009) [arXiv:0810.2959 [hep-ph]].

[37] H. C. Ahn, D. K. Hong, C. Park and S. Siwach, "Spin 3/2 Baryons and Form Factors in AdS/QCD," Phys. Rev. D 80, 054001 (2009) arXiv:0904.3731 [hep$\mathrm{ph}]$.

[38] Y. Q. Sui, Y. L. Wu, Z. F. Xie and Y. B. Yang, "Prediction for the Mass Spectra of Resonance Mesons in the Soft-Wall AdS/QCD with a Modified 5D Metric," arXiv:0909.3887 [hep-ph].

[39] M. Kirchbach and C. B. Compean, "High Spin Baryons In Quantum Mechanical Chromodynamics," AIP Conf. Proc. 1116 (2009) 334.

[40] The SU(6) 70-plet of light baryons is not well described in this simple model, and further investigation is necessary. See: [36].

[41] E. Klempt and A. Zaitsev, "Glueballs, Hybrids, Multiquarks. Experimental facts versus QCD inspired concepts," Phys. Rept. 454, 1 (2007) arXiv:0708.4016 [hep$\mathrm{ph}]]$. 